\title{
Construcción de triángulos con materiales manipulativos
}

\author{
Mª Carmen Fernández Molinero y Aurora del Río Cabeza. Universidad de Granada
}

\begin{abstract}
Recepción: 11 de diciembre de 2015 | Revisión: 20 de diciembre de 2015 | Aceptación/Publicación: 21 de diciembre de 2015 Correspondencia: mcarmenfm@correo.ugr.es | adelrio@ugr.es

Citar: Fernandez-Molinero, MC. y del Rio Cabeza, A. (2015). Construcción de triángulos con materiales manipulativos. ReiDoCrea, 4, 386-390.
\end{abstract}

Resumen: La enseñanza de la geometría presenta dificultades de aprendizaje en Educación Primaria que induce a frecuentes errores en Matemáticas. Por esta razón, este trabajo se basa en la descripción de tareas usando materiales manipulativos, con el objetivo de proporcionar al alumnado un acercamiento sencillo y familiar a los conceptos Geométricos. Por lo tanto en este estudio de investigación se presenta la construcción de triángulos para subsanar algunos errores y dificultades conceptuales que aparecen ligados a aprendizaje de la Geometría en Educación Primaria.

Palabras clave: Material Didáctico | Geometría

Building Triangles with Educational Materials

Abstract: The teaching of Geometry has learning difficulties in Primary Education which leads to frequent mistakes in Math. Therefore, this work is based on the job description using manipulative materials, with the aim of providing students with a simple and familiar approach to geometric concepts. Therefore in this research the construction of triangles is presented to correct some errors and conceptual difficulties appear related to Geometry learning in Primary Education.

Key words: Teaching Materials | Geometry

Este estudio de investigación es parte del Trabajo Fin de Grado de Educación Primaria de la Universidad de Granada, titulado: Estudio de la Geometría del triángulo con pajitas (Fernández-Molinero, 2015).

\section{Introducción}

En el currículo escolar donde en el alumnado aprende a razonar y a ver la estructura axiomática de las Matemáticas es la Geometría (NCTM, 2003). El trabajo de desarrollo de habilidades de razonamiento y de justificación de propiedades, que se inicia en la etapa de Educación Primaria, concluye en la etapa de Secundaria. Si en la etapa inicial de desarrollo utilizamos un material manipulativo que facilite y contribuya al desarrollo de la capacidad de visualización, contribuiremos al desarrollo del razonamiento y de las habilidades para justificar cualquier supuesto, y por ende a la mejora del sentido espacial (Flores y Rico, 2015).

El estudio de la Geometría se inicia con la geometría plana, y dentro de ella, uno de los focos más importantes es el estudio de los polígonos. El polígono más simple de todos es el triángulo, y además es base para el estudio posterior de los demás polígonos, de ahí su importancia y la necesidad de que su estudio y comprensión en profundidad (Castro, 2001).

Un triángulo es una figura geométrica plana que está constituida por tres lados y tres vértices. Como paso previo al estudio del triángulo, necesitamos definir sus elementos básicos. Para algunos de estos elementos básicos (recta y punto) recurriremos a las definiciones que se recogen en el libro de "Los elementos" de Euclídes:

- Punto: un punto es lo que no tiene partes.

- Recta: es una longitud sin anchura. 
- Segmento: es la parte de la recta delimitada por dos de sus puntos A y B (Segovia y Rico, 2011).

- Ángulo: región delimitada por dos semirrectas con el punto de inicio de las semirrectas en común (Castro, 2001).

En el caso del triángulo, estos elementos básicos se suelen denominar vértice, lado y ángulo. Para poder construir un triángulo necesitaremos conocer alguno de sus elementos básicos, así se deberán cumplir una serie de condiciones para que en función de los elementos básicos de los que dispongamos:

- Conocidos tres lados, siempre y cuando estos cumplan la que se conoce como la desigualdad triangular (la suma de dos lados cualesquiera es mayor el tercero).

- Conocidos dos lados y el ángulo comprendido entre ellos.

- Conocidos dos ángulos y el lado en común.

Diversos autores como Flores y Rico (2015) y Van de Walle (2001) recogen una serie de errores y dificultades que aparecen ligadas al bloque de geometría. Entre estos errores y dificultades encontramos el uso de polígonos abiertos a la hora de construir un triángulo y el uso de líneas curvas en la construcción de polígonos. Un material manipulativo adecuado contribuirá a evitar y corregir errores, ya que los propios alumnos serán los que realicen sus construcciones.

Utilizar un material como apoyo para el aprendizaje contribuye a activar el conocimiento debido a que el aprendizaje que se consigue es más significativo, dado que es importante considerar lo que el alumno ya sabe de tal manera que establezca relación con aquello que debe aprender, puesto que se produce una interacción entre los conocimientos ya adquiridos y la nueva información. El conocimiento como acción implica que una persona reúne, descubre o crea conocimiento en el curso de alguna actividad que tiene una finalidad (Romberg, 1991). Por otra parte, acciones como observar o manipular facilitan el desarrollo de capacidades geométricas (NCTM, 2003).

Flores, Lupiañez, Berenguer ,Marín y Molina (2011) recogen las características que debe cumplir un material manipulativo adecuado:

- Disponibilidad en el momento que se decide usar.

- Equipamiento suficiente para todos los alumnos y alumnas.

- Cierta práctica por parte del docente y de los alumnos y alumnas en el manejo antes de empezar a razonar matemáticamente con ellos.

- Temporalización adecuada que permita extraer consecuencias a la mayoría de los alumnos y alumnas en los momentos previstos.

La construcción de un concepto constituye una combinación de procesos visuales y analíticos que debe ser organizada mediante una serie de tareas de complejidad creciente. Dichas tareas focalizarán su demanda en el uso de un vocabulario correcto, consiguiendo que éstos hagan un uso correcto de los conceptos para comunicar sus ideas. (Flores y Rico, 2015).

Las construcciones erróneas en Matemáticas pueden aprovecharse didácticamente en el aprendizaje, así mediante las cuestiones pertinentes, podrá detectarse si los errores son debidos a la construcción o al propio concepto de triángulo que tenga el alumno. 


\section{Método}

\section{¿Cómo construimos los triángulos con pajitas y plastilina?}

En este apartado describimos los materiales y cómo realizar las construcciones de los distintos tipos de triángulos.

\section{a) Definición de los materiales}

Los materiales que proponemos para trabajar la geometría del triángulo, son pajitas y plastilina. Así que se define:

- Punto: una bola de plastilina.

- Recta: una pajita.

- Segmento: dos bolas de plastilina en los extremos de una pajita.

- Ángulo: dos pajitas unidas por una bola de plastilina.

La bola de plastilina, al igual que el punto, se encuentra en un plano e indica posición. La utilizaremos para ver dónde se cortan dos rectas y dónde empieza un ángulo. Por otra parte, las pajitas nos van a ayudar a delimitar el plano, formar líneas, ángulos... también nos permiten cortarlas fácilmente para conseguir el tamaño que necesitamos para trabajar. A partir de este momento empezamos con la construcción de triángulos, según los datos que conozcamos, tal y como describíamos en la introducción.

\section{b) Construcción de triángulos}

Conocidos los tres lados:

- Si tenemos los tres lados, tan solo tenemos que trasladar las medidas a las pajitas, recortarlas y pegarlas por los extremos. Si las medidas dadas cumplen con la conocida como desigualdad triangular, que dice que la suma de dos lados es mayor que la medida del tercero, podremos construir nuestro triángulo.

Figuras 1. Construcción de un triángulo conocidos 3 lados

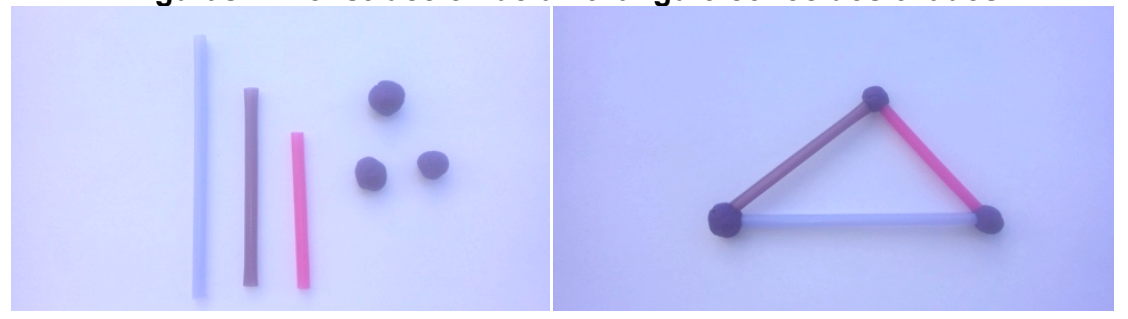

- Conocidos dos lados y un ángulo comprendido entre ellos:

1. Si tenemos dos lados y el ángulo comprendido entre ellos, el tercer lado se obtiene como unión de los vértices libres de los lados conocidos.

2. Cortamos dos pajitas según la medida de los lados que nos han dado y con ellas creamos el ángulo que nos dan. El ángulo lo construiremos colocando las pajitas sobre las semirrectas que definen el ángulo dibujado sobre el papel. 
3. Una vez construido el ángulo con las pajitas, colocamos la tercera pajita con la medida necesaria para poder completar el triángulo. Esta medida es única.

Figuras 2. Construcción de un triángulo conocidos dos lados y un ángulo

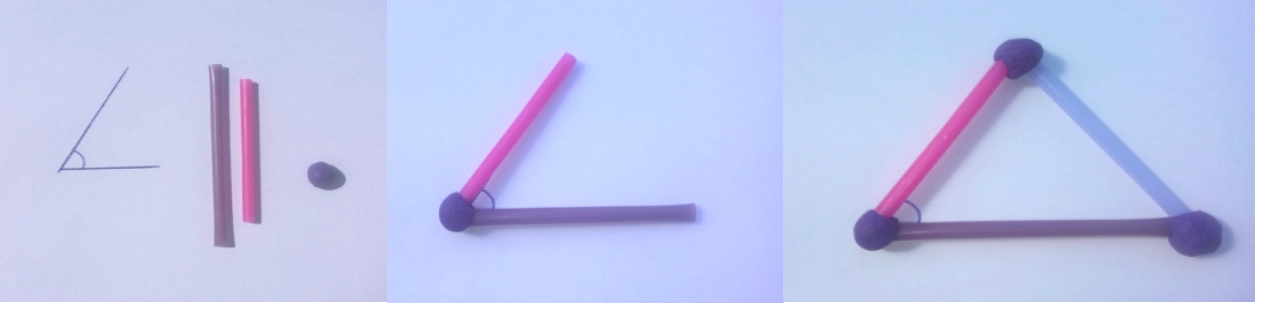

- Conocidos dos ángulos y el lado común:

1. Si tenemos dos ángulos y la medida del lado que los une tan solo tendremos que alargar las semirrectas no comunes que definen los ángulos hasta que se corten. El punto donde se cortan determinará el tercer vértice del triángulo.

2. Cortamos una pajita con la medida del lado que nos dan y sobre los otros lados del ángulo situamos pajitas con el tamaño suficiente. Utilizaremos unas pajitas auxiliares para construir los ángulos, que luego se sustituirán por el lado conocido, también utilizaremos el papel para trasportar los ángulos. Cortaremos estas pajitas en el punto de intersección y las uniremos con una bola.

Figuras 3. Construcción de un triángulo conocidos dos ángulos y el lado común

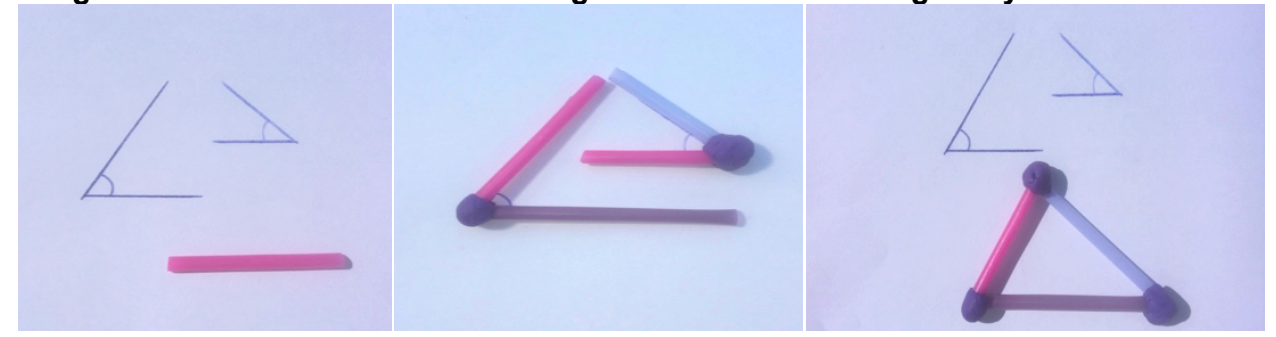

\section{Conclusiones}

Con este trabajo pretendemos acercar una parte de la geometría a los alumnos mediante el uso de un material adaptado a sus distintas capacidades y niveles de conocimientos previos. Las pajitas y la plastilina son materiales manipulativos que ayudan a entender los contenidos, gracias a que podemos tocarlos, romperlos, arreglarlos y colocarlos en diferentes posiciones.

Si atendemos a las características propuestas por Flores, Lupiañez, Berenguer, Marín y Molina (2011), vemos que nuestro material se ajusta a todas ellas. Así, se trata de un material cotidiano, de fácil adquisición y que por ello puede estar disponible en el momento que se decide usar, pudiendo incluso el alumno hacer uso de él en su propia casa, una vez concluida la instrucción. En cuanto al equipamiento, claramente es suficiente, por esa facilidad de disponer de él y además no necesita unas infraestructuras especiales por lo que se puede usar en el aula de clase. Con respecto a la práctica en el manejo, incidimos en la idea anterior, es un material muy familiar tanto para el docente como para los alumnos, y que no requiere unos conocimientos y manejo especiales. Finalmente, consideramos que la temporalización de las tareas es flexible y por tanto adecuada a que cada alumno pueda extraer las consecuencias 
previstas. La flexibilidad en la temporalización se debe, entre otras cosas, a la facilidad para poder trabajar este material en casa.

Consideramos las pajitas y la plastilina, como material didáctico manipulativo, que proporciona una mayor implicación del alumno en las tareas a realizar ya que la manipulación "constituye un modo de dar sentido al conocimiento matemático" (Segovia y Rico, 2001).

Por otra parte, estos materiales son incentivos, ya que facilitan el aprendizaje y fomentan el interés. Además, al construir ellos mismos los distintos triángulos hacen uso de las propiedades de los triángulos, contribuyendo al mejor aprendizaje y comprensión de las mismas, al verlas representadas delante de ellos.

El uso de estos materiales permite la manipulación de los objetos geométricos, un acercamiento intuitivo a la geometría del plano y los procesos de construcción implicados son lógicos y eficientes.

Este material nos permite además subsanar algunos errores como son la confusión con líneas poligonales abiertas o cerradas, ya que el alumno deberá "enganchar" la pajita con la bola de plastilina y por lo tanto no van a quedar contornos abiertos. Además, la rigidez de las pajitas evitará construir líneas curvas.

Finalmente, nos permitirá incidir en la importancia de la desigualdad triangular, ya que si les diéramos a los alumnos tres pajitas cualesquiera que no cumplieran esta desigualdad, la rigidez del material no permitiría construir un triángulo. Éstos son ejemplos de errores en la construcción de triángulos.

Así, en este trabajo hemos visto que partiendo de los elementos básicos, lado y ángulo podemos construir un triángulo. Hemos empezado con 3 lados, después 2 lados y un ángulo y después 1 lado y dos ángulos. La siguiente posibilidad, si siguiéramos esta serie sería 3 ángulos. Si propusiéramos a los alumnos construir un triángulo conocidos los 3 ángulos, podrán ver que cada uno de ellos obtiene triángulos distintos, de forma, que surge de manera natural el concepto de triángulos semejantes. Este concepto permitirá introducir un resultado tan importante en las matemáticas, como es el Teorema Thales.

Referencias

Castro, E. (2001). Didáctica de la matemática en la Educación Primaria. Madrid: Síntesis.

Fernández-Molinero, MC. (2015). Estudio de la geometría del triángulo con pajitas. Granada: Universidad de Granada.

Flores, P., Lupiáñez, JL., Berenguer, L., Marín, A. y Molina, M. (2011). Materiales y recursos en el aula de matemáticas. Granada: Departamento de Didáctica de la Matemática de la Universidad de Granada.

Flores, P. y Rico, L. (2015). Enseñanza y aprendizaje de las matemáticas en educación primaria. Madrid: PIRÁMIDE.

NCTM (2003). Principios y estándares para la Educación Matemática. Granada: SAEM THALES.

Romberg, TA. (1991). Características problemáticas del currículo escolar de matemáticas. Revista de Educación, 294, 327-330.

Segovia, I. y Rico, L. (2011). Matemáticas para maestros de Educación Primaria. Madrid: Pirámide.

Van de Walle, J.A. (2001). Elementary and Middle School Mathematics: Teaching Developmentally. New York: Longman. 